\title{
On Impact Damage Detection and Quantification for CFRP Laminates using Structural Response Data Only.
}

\begin{abstract}
The overall purpose of the research is to detect and attempt to quantify impact damage in structures made from composite materials. A study that uses simplified coupon specimens made from a Carbon Fibre-Reinforced Polymer (CFRP) prepreg with 11, 12 and 13 plies is presented. PZT sensors were placed at three separate locations in each test specimen to record the responses from impact events. To perform damaging impact tests, an instrumented droptest machine was used and the impact energy was set to cover a range of $0.37-41.72 \mathrm{~J}$. The response signals captured from each sensor were recorded by a data acquisition system for subsequent evaluation. The impacted specimens were examined with an X-ray technique to determine the extent of the damaged areas and it was found that the apparent damaged area grew monotonically with impact energy. A number of simple univariate and multivariate features were extracted from the sensor signals recorded during impact by computing their spectra and calculating frequency centroids. The concept of discordancy from the statistical discipline of outlier analysis is employed in order to separate the responses from nondamaging and damaging impacts. The results show that the potential damage indices introduced here provide a means of identifying damaging impacts from the response data alone.
\end{abstract}

Keyword: Carbon fibre; Prepreg; Impact behaviour; Mechanical testing; Signal processing; Outlier analysis 